\title{
Methylation status and protein expression of RASSF1A in endometriosis
}

\author{
YU WU ${ }^{1}$, MINGDE ZHANG ${ }^{2}$, XIAN ZHANG ${ }^{1}$, ZHENZHOU XU $^{1}$ and WEIGUO JIN ${ }^{1}$ \\ ${ }^{1}$ Department of Gynecology and Obstetrics, Liaocheng People's Hospital, \\ Liaocheng Clinical School of Taishan Medical University, Liaocheng, Shangdong 252000; \\ ${ }^{2}$ Department of Ear and Throat, Dongchang People's Hospital, Liaocheng, Shangdong 252000, P.R. China
}

Received May 24, 2014; Accepted April 5, 2016

DOI: $10.3892 / \mathrm{ol} .2016 .4512$

\begin{abstract}
Ras association domain family 1A (RASSF1A) gene inactivation by promoter hypermethylation is a common event in the development of a variety of types of human cancer. Accumulated evidence has demonstrated that DNA methylation serve a critical role in the pathogenesis of endometriosis. The aim of the present study was to analyze the methylation status and protein expression of RASSF1A in endometriosis (EMS). The ectopic and corresponding eutopic endometrium tissues were collected from 45 women with EMS (EMS group) and normal endometrium tissues from 20 women without EMS (control group). The methylation status of RASSF1A was examined by methylation specific polymerase chain reaction (MSP). Immunohistochemistry was performed to measure RASSF1A protein level in endometrium tissues. In normal endometrium samples, RASSF1A protein expression was significantly higher at the secretory phase than the proliferative phase. RASSF1A protein expression in the ectopic endometrium tissues and eutopic endometrium tissues were significantly reduced than in normal endometrium $(\mathrm{P}<0.05)$. The frequency of aberrant methylation of RASSF1A was $55.56 \%$ in ectopic endometrium and $33.33 \%$ in paired eutopic endometrium, whereas such methylation was not detected in normal endometrium. Moreover, RASSF1A promoter hypermethylation was frequently associated with reduced expression of RASSF1A, and was common in advanced stage in ectopic endometrium of EMS. Epigenetic inactivation of RASSF1A through aberrant promoter methylation may be important in the formation and progression of EMS, and assessment of RASSF1A methylation status in eutopic endometrium may be a potentially useful biomarker to enhance the early detection of EMS.
\end{abstract}

Correspondence to: $\mathrm{Dr} \mathrm{Yu} \mathrm{Wu}$, Department of Gynecology and Obstetrics, Liaocheng People's Hospital, Liaocheng Clinical School of Taishan Medical University, 67 Dongchang West Road, Liaocheng, Shangdong 252000, P.R. China

E-mail: liumangyu2003@163.com

Key words: endometriosis, RASSF1A, hypermethylation, ectopic endometrium, eutopic endometrium

\section{Introduction}

Endometriosis (EMS), a common, benign, estrogen-dependent disease affecting $5-10 \%$ of women of reproductive age, is characterized by the presence of ectopic endometrial glands and stroma outside of the uterine cavity, primarily in the peritoneum, ovaries and rectovaginal septum (1). Women suffering EMS may present with chronic pelvic pain, dysmenorrhea, dyspareunia and/or infertility. The incidence has been on the rise over the years (2). A number of theories, such as Sampson's theory (3), have been proposed to explain the etiology of EMS, but the exact mechanism of its development remains unknown.

The novel candidate tumor suppressor the Ras association domain family 1A (RASSF1A) gene has been recently identified and mapped to chromosome 3p21.3 (4). It was previously demonstrated that RASSF1A gene inactivation by promoter hypermethylation is a common event in the development of a variety of types of human cancer, for example in the lung, breast, ovary, endometrial and cervical carcinomas (5-7). The role of RASSF1A as a tumor suppressor gene has been suggested since exogenous expression of RASSF1A decreases in vitro-colony formation, suppresses anchorage-independent growth, and reduces the tumorigenicity of cancer cells in vivo $(4,8)$.

EMS is a benign gynecological disease which displays malignant behaviors, such as enhanced proliferation and cell invasion, ectopic implantation of distant organs similar to tumor metastasis (9). Accumulating evidence has indicated that DNA methylation serves definite roles in the pathogenesis of EMS $(10,11)$. It is therefore reasonable to hypothesize that epigenetic inactivation of RASSF1A may have a role in EMS. The aim of the present study was to investigate RASSF1A protein expression and the methylation status in the ectopic and eutopic endometrium of fertile women with EMS and normal endometrium of women without EMS by immunohistochemistry and methylation specific polymerase chain reaction (MSP) to better define the possible involvement of RASSF1A in the pathogenesis of EMS.

\section{Materials and methods}

Patients and samples. After informed consent was obtained, ectopic endometrium from the cyst walls of ovarian EMS 
and the matched eutopic endometrium from the uterus were taken from 45 patients, aged 24-50 years. EMS stages were confirmed according to the Revised American Fertility Society Classification (12). For controls, normal endometrium samples were obtained from 20 healthy women, aged 34-48 years, who underwent tubal sterilization that was confirmed laparoscopically to be free of EMS. All of the patients underwent surgical treatment in the Department Gynecology and Obstetrics at Liaocheng People's hospital (Shangdong, China) between February 2012 and December 2013. None of these patients had received any gonadotropin-releasing hormone analogue, antibiotics, radio-, chemo-, or hormone therapy in the last 6 months prior to the surgery. All samples were histologically confirmed, and the phase of the menstrual cycle was determined by preoperative history and histologic examination. The mean ages of subjects were $41 \pm 2$ years in the experimental group, and $38 \pm 2$ years in the control group, and there were no significant differences between the two groups with respect to age. Each biopsy was divided in two fragments, which were either frozen in liquid nitrogen (and stored in $\mathrm{a}-80^{\circ} \mathrm{C}$ for DNA extraction) or fixed in $10 \%$ buffered formalin for histological confirmation of endometriosis and immunohistochemistry. The study was approved by the Ethics Committee of Liaocheng Hospital.

Immunohistochemistry. The expression of RASSF1A protein was evaluated by immunohistochemistry which was performed with a standard streptavidin-peroxidase (S-P) technique, using diamino-benzidine (DAB) as a chromogen. The procedures were as follows: tissue sections were dewaxed in xylene and gradient alcohol with decreasing concentrations. Antigen retrieval was performed using microwave irradiation at $750 \mathrm{~W}$ for $15 \mathrm{~min}$ in $10 \mathrm{mM}$ citrate buffer ( $\mathrm{pH} \mathrm{6.0).} \mathrm{After} \mathrm{treatment}$ with $3 \% \mathrm{H}_{2} \mathrm{O}_{2}$ for $15 \mathrm{~min}$, and $5 \%$ normal milk (Solarbio, China) for $30 \mathrm{~min}$ to block nonspecific protein binding. The slide were then incubated over night with anti-RASSF1A (mouse monoclonal IgG, clone 3F3, code number AB 23950, Santa Cruz Biotechnology, Dallas, TX, USA; 1:500 dilution). After 3 washes in PBS, sections were incubated with goat anti-mouse IgG-HRP (sc-2031, Santa Curz Biotechnology, Dallas, TX, USA; 1:1,000 dilution) in a streptavidin-peroxidase reagent (ThermoFisher Scientific, Inc., Waltham, MA, USA) for $15 \mathrm{~min}$, then the peroxidase reaction was initiated by incubating tissue sections in DAB reagent (Beyotime Insititute of Biotechnology, Inc., Shanghai, China) for 10-20 min at room temperature. Sections were washed 3 times in distilled water. Slides were finally counterstained with Mayer hematoxylin (Beyotime Insititute of Technology, Inc.).

Brown staining was considered positive. Sections known to express RASSF1A were used as the positive control, and PBS was used as the negative control. RASSF1A protein expression appeared in the cytoplasm of the cells. Sections were observed under an optical microscope (Nikon, Tokyo, Japan) in x40 magnification. A total of 5 visual fields were randomly selected in each section, and they were graded at 4 levels according to the percentage of positively stained cells: negative $(-),<5 \%$; positive $(+), 5-25 \%$; positive $(++), 25-50 \%$; and positive $(+++),>50 \%$.

DNA extraction and bisulfite modification. DNA was extracted from frozen preserved tissues by proteinase $\mathrm{K}$ digestion (Beyotime Insititute of Biotechnology, Inc.) and a phenol/chloroform extraction method (Beyotime Insititute of Biotechnology, Inc.) (13), denatured with $\mathrm{NaOH}$ and treated with sodium bisulphate as previously described (14).

Methylation specific polymerase chain reaction (MSP) for RASSF1A. Promoter hypermethylation of the RASSF1A gene was determined by the MSP as previously described (13). PCR was performed using the primer sequences of RASSF1A listed below: The methylated reaction primer sequences were 5'-GTGTTAACGCGTTGCGTATC-3' (sense) and 5'-AACCCCGCGAACTAAAAACGA-3' (antisense), and the produce size was $93 \mathrm{bp}$. For the unmethylated reaction, the primer sequences were 5'-TTTGGTTGGAGTGTGTTAATGTG-3' (sense) and 5'-CAAACCCCACAAACTAAAAACAA-3' (antisense), and the produce size was $103 \mathrm{bp}$. Primers and PCR reagents were purchased from Shanghai Sangon Biological Engineering Technology (Shanghai, China). The PCR parameters was as follows: $94^{\circ} \mathrm{C}$ for $10 \mathrm{~min} ; 40$ amplification cycles of $30 \mathrm{sec}$ at $94^{\circ} \mathrm{C}, 30 \mathrm{sec}$ at the annealing temperature $\left(59^{\circ} \mathrm{C}\right), 30 \mathrm{sec}$ at $72^{\circ} \mathrm{C}$, and finally $72^{\circ} \mathrm{C}$ for $10 \mathrm{~min}$. Products were loaded onto $2 \%$ agarose gels (Santa Cruz Biotechnology, Inc.), stained with ethidium bromide (Beyotime Insititute of Biotechnology, Inc.) and visualized under ultraviolet illumination.

Statistical analysis. All statistical analysis were performed using the SPSS software, Version 13.0 (SPSS, Inc., Chicago, IL, USA). $\chi^{2}$-test and Fisher's exact were used to compare RASSF1A protein expression and methylation status between the cases, and with menstrual cycle phase and clinical stage. Pearson's correlation analysis was used to evaluate the relationship between RASSF1A protein expression and methylaion status in EMS. $\mathrm{P}<0.05$ was considered to indicate a statistically significant difference.

\section{Results}

Cycle phase. All surgeries were performed during the proliferative and secretory phase in 2 groups. In EMS group, surgery was performed during the proliferative phase in 21 patients and during the secretory phase in 24 patients. In the control group, surgery was performed during the proliferative phase in 5 patients and during the secretory phase in 15 patients. There were no significant differences between the 2 groups with cycle phase $\left(\chi^{2}=2.708, P=0.100\right)$.

The RASSF1A protein expression in different endometrium tissues. To determine RASSF1A protein expression in EMS, ectopic and eutopic endometrium samples from 45 patients with EMS and 20 controls without EMS were examined by immunohistochemistry. Immunohistochemical results showed that RASSF1A was mainly expressed in the cytoplasm of glandular epithelial cells in the endometrial tissues.

In normal endometrium samples, immunohistochemistry showed that RASSF1A protein expression was significantly higher at the secretory phase than at the proliferative phase $(\mathrm{P}<0.05)$. Either in ectopic or eutopic endometrium samples, the protein level of RASSF1A at the secretory phase was higher than at the proliferative phase, although this was not significant $(\mathrm{P}>0.05)$. The results are presented in Table I. 
Table I. The protein expression of the RASSF1A in both ectopic and eutopic endometrium of EMS and normal endometrium of non-EMS and the relationship with the menstrual cycle phase.

\begin{tabular}{|c|c|c|c|c|c|c|c|}
\hline \multirow[b]{2}{*}{ Groups } & \multirow[b]{2}{*}{$\mathrm{n}$} & \multicolumn{4}{|c|}{ RASSF1A protein expression } & \multirow[b]{2}{*}{$\chi^{2}$-value } & \multirow[b]{2}{*}{ P-value } \\
\hline & & - & + & ++ & +++ & & \\
\hline Ectopic endometrium of EMS & 45 & 28 & 11 & 6 & 0 & & \\
\hline Proliferative phase & 21 & 15 & 4 & 2 & 0 & 1.420 & 0.233 \\
\hline Secretory phase & 24 & 13 & 7 & 4 & 0 & & \\
\hline Eutopic endometrium of EMS & 45 & 18 & 17 & 9 & 1 & & \\
\hline Proliferative phase & 21 & 11 & 7 & 3 & 0 & 2.515 & 0.113 \\
\hline Secretory phase & 24 & 7 & 10 & 6 & 1 & & \\
\hline Normal Endometrium of non-EMS & 20 & 3 & 4 & 7 & 6 & & \\
\hline Proliferative phase & 5 & 3 & 1 & 1 & 0 & & 0.009 \\
\hline Secretory phase & 15 & 0 & 3 & 6 & 6 & & \\
\hline
\end{tabular}

EMS, endometriosis; RASSF1A, Ras association domain family 1A.

Table II. The promoter hypermethylated status of the RASSF1A gene in ectopic and eutopic endometrium of EMS and the relationship with the menstrual cycle phase and clinical stages.

RASSF1A promoter methylation

\begin{tabular}{|c|c|c|c|c|c|}
\hline & & & & & \\
\hline Groups & $\mathrm{n}$ & $\mathrm{M}$ & $\mathrm{U}$ & $\chi^{2}$-value & P-value \\
\hline Ectopic endometrium of EMS & 45 & 25 & 20 & & \\
\hline Proliferative phase & 21 & 14 & 7 & 1.969 & 0.161 \\
\hline Secretory phase & 24 & 11 & 13 & & \\
\hline Eutopic endometrium of EMS & 45 & 15 & 30 & & \\
\hline Proliferative phase & 21 & 8 & 13 & 0.402 & 0.526 \\
\hline Secretory phase & 24 & 7 & 17 & & \\
\hline Clinical stages of EMS ${ }^{a}$ & 45 & 25 & 20 & & \\
\hline Stage I, II & 16 & 5 & 11 & 5.940 & 0.015 \\
\hline Stage III, IV & 29 & 20 & 9 & & \\
\hline Clinical stages of EMS ${ }^{b}$ & 45 & 15 & 30 & & \\
\hline Stage I, II & 16 & 11 & 5 & 1.751 & 0.186 \\
\hline Stage III, IV & 29 & 14 & 15 & & \\
\hline
\end{tabular}

ain ectopic endometrium, bin eutopic endometrium. EMS, endometriosis; RASSF1A, Ras association domain family $1 \mathrm{~A}$.
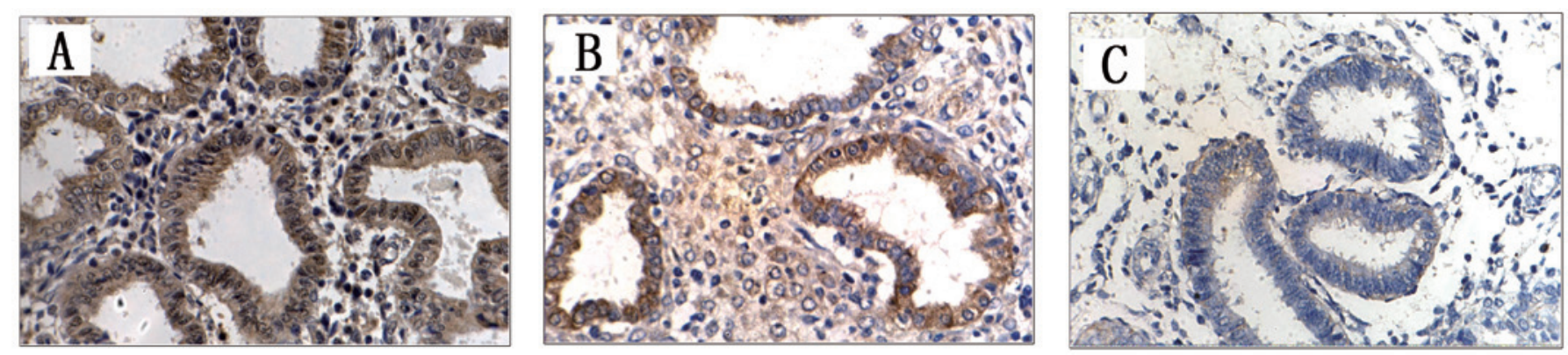

Figure 1. RASSF1A protein expression was assessed in different endometrium samples by immunohistochemistry. (A) A case of normal endometrium tissues from a woman without EMS demonstrating strong RASSF1A immunoreactivity. (B) A case of ectopic endometrium tissues from a women with EMS demonstrating moderate levels of RASSF1A immunoreactivity. (C) A case of eutopic endometrium tissues from a woman with EMS demonstrating weak RASSF1A immunoreactivity. EMS, endometriosis; RASSF1A, Ras association domain family 1A. 
Table III. Correlation between the protein expression and methylation of RASSF1A in ectopic and eutopic endometrium of EMS .

\begin{tabular}{|c|c|c|c|c|c|c|}
\hline & \multirow[b]{2}{*}{ RASSF1A } & \multicolumn{5}{|c|}{ RASSF1A promoter methylation } \\
\hline & & M & $\mathrm{U}$ & $\chi^{2}$-value & $\mathrm{r}$ & P-value \\
\hline \multicolumn{7}{|l|}{ Ectopic endometrium of EMS } \\
\hline & + & 3 & 14 & 15.901 & -0.594 & 0.000 \\
\hline & - & 22 & 6 & & & \\
\hline \multicolumn{7}{|l|}{ Eutopic endometrium of EMS } \\
\hline & + & 3 & 24 & 15 & -0.577 & 0.000 \\
\hline & - & 12 & 6 & & & \\
\hline
\end{tabular}

EMS, endometriosis; RASSF1A, Ras association domain family $1 \mathrm{~A}$.
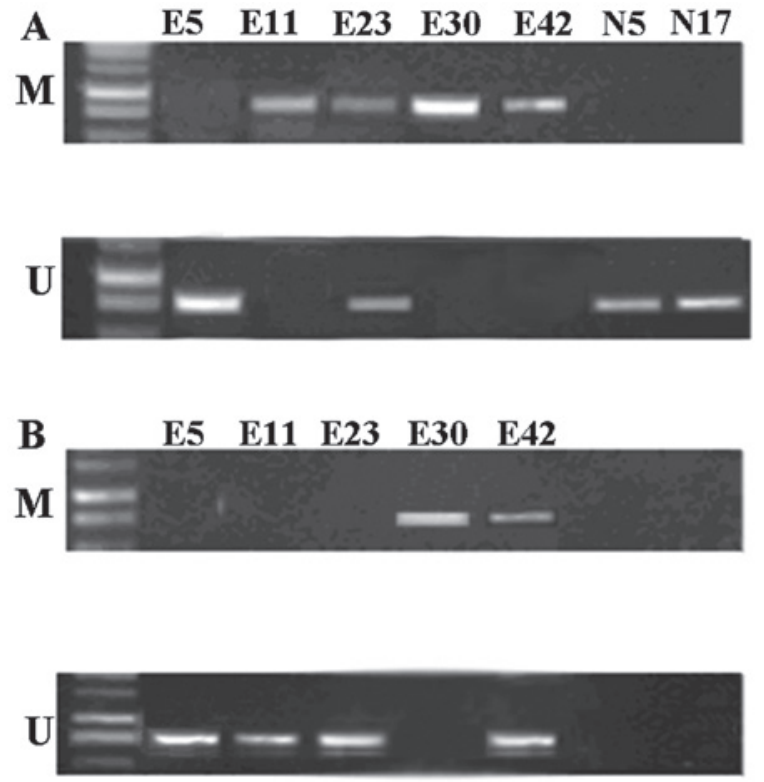

Figure 2. RASSF1A promoter hypermethylation status in EMS by MSP. (A) Promoter hypermethylation status of the RASSF1A gene in ectopic endometrium tissues.(B) Promoter hypermethylation status of the RASSF1A gene in eutopic endometrium tissues. M, MSP; U, unmethylated-specific PCR. E, endometriosis; N, normal endometrium samples. EMS, endometriosis; PCR, polymerase chain reaction; MSP, methylation specific PCR; RASSF1A, Ras association domain family $1 \mathrm{~A}$

Fig. 1 demonstrated different degrees of RASSF1A protein expression in the 3 groups. The positive expression rates of RASSF1A in the endometrial tissues of each group were calculated and compared (Table I). The rates were $37.78 \%(17 / 45)$ and $60 \%$ (27/45) in the EMS ectopic and eutopic endometrium samples, respectively. In the control group, the positive expression rate was $85 \%(17 / 20)$. The expression level of RASSF1A was significantly lower in ectopic endometrium $\left(\chi^{2}=12.377\right.$, $\mathrm{P}<0.0001)$ and eutopic endometrium $\left(\chi^{2}=3.957, \mathrm{P}=0.047\right)$ as compared with control subjects. The difference between ectopic and eutopic endometrium also was statistically significant $\left(\chi^{2}=4.447, \mathrm{P}=0.035\right)$.

Methylation status of RASSF1A in different endometrial samples. To determine the correlation between DNA methylation and RASSF1A protein expression in endometrial tissues, the methylation status of RASSF1A was evaluated in the above three endometrial tissues by using MSP. The results demonstrated that the promoter was hypermethylated in $55.56 \%(25 / 45)$ of RASSF1A genes in ectopic endometrium and $33.33 \%(15 / 45)$ in eutopic endometrium. In contrast, no RASSF1A promoter hypermethylation was observed in the control subjects. The difference was statistically significant between the above three groups $(\mathrm{P}<0.05)$. Thus, the RASSF1A promoter hypermethylation occurred exclusively in EMS. Representative examples are presented in Fig. 2.

As shown in Table II that frequency of promoter hypermethylation of RASSF1A did not vary significantly throughout the menstrual cycle either in ectopic endometrium or in eutopic endometrium.

The EMS stages were between stage I to IV. There were 16 cases of stage I and II, 29 cases of stage III and IV. As Table III demonstrates, in the ectopic endometrium, the frequency of promoter hypermethylation of RASSF1A at advanced stage (stage III, IV) was significantly higher than at early stage (stage I, II) $(\mathrm{P}<0.05)$. However, in the eutopic endometrium, frequency of promoter hypermethylation of RASSF1A at different clinical stages was not significantly different.

Correlation between RASSF1A protein expression and promoter hypermethylation. Finally, the correlation between RASSF1A immunoexpression and the methylation status in EMS was determined. In the ectopic endometrium, RASSF1A was methylated in 22 of 28 cases and negative for RASSF1A immunostaining. Meanwhile, in eutopic endometrium, RASSF1A protein expression was dramatically reduced in cases with RASSF1A promoter mythylation as compared with unmethylated cases. There was generally a negative correlation between hypermethylated RASSF1A and protein expression in both ectopic endometrium and eutopic endometrium. These data suggest that hypermethylation of RASSF1A promoter may be associated with abnormally low or absent expression in EMS. The results are shown in Table III.

\section{Discussion}

Epigenetics refers to heritable changes in DNA and chromatin that impact gene expression without changes in DNA sequence (15). There are 2 basic epigenetic regulatory 
mechanisms: DNA methylation and histone modifications. DNA methylation is the best understood and most extensively studied epigenetic mechanism and refers to the covalent modification of post-replicative DNA, when a methyl group is added to the cytosine ring to form methyl cytosine (16). DNA methylation serves a critical role in the regulation of gene expression in development, differentiation, and complex diseases, cancer being the most prominent example $(15,16)$. EMS shares some common features with cancer. For instance, the loss of the tumor suppressor PTEN in endometrial cancer was well-established in 2010 (17) and decreased PTEN expression has also been reported in the eutopic and ectopic endometrial of patients with EMS as well (18).

In women of reproductive age, EMS mainly involves the ovary (2); an endometriotic cyst of ovary is the most common type of EMS. Ovarian EMS was selected as the focus of the present study. Meanwhile, patients who underwent tubal sterilization that were confirmed to be laparoscopically to be free of EMS were selected as the control group.

The present study investigated the protein expression of RASSF1A in EMS. RASSF1A is localized in the cytoplasm of glandular epithelial cells in both ectopic and eutopic endometrium of EMS patients and in normal endometrium from women with non-EMS. It was demonstrated that RASSF1A was stronger expressed in normal endometrium. This is similar to Pallarés et al (19). In the present study, RASSF1A protein expression was significantly higher at the secretory phase than at the proliferative phase, but this is different from Pallarés et al (19), who found RASSF1A immunoexpression was significantly higher in the proliferative phase than in the secretory endometrium. This difference may be due in part to different patients and agents used.

In the present study, experimental evidence was presented that indicated that the frequency of methylation of the RASSF1A gene in ectopic endometrium and eutopic endometrium were 55.56 and $33.33 \%$, respectively, whereas such methylation was not detected in normal endometrium. Combined with the immunohistochemical results, the positive expression rate of RASSF1A protein in the ectopic and eutopic endometrium were lower than in normal endometrium. In addition, a clear inverse relationship was observed between the extent of methylation in the RASSF1A promoter $\mathrm{CpG}$ island and its protein levels, either in ectopic or in eutopic endometrium. In addition, endometrium with RASSF1A methylation exhibited weak or lost RASSF1A protein expression in comparison to endometrium with RASSF1A unmethylation. Taken together, these results provide strong evidence that RASSF1A hypermethylation may constitute the mechanism through which RASSF1A expression is downregulated in endometriosis. Epigenetic inactivation of RASSF1A through aberrant promoter methylation may serve an important role in the pathogenesis of EMS.

The present data also demonstrated that there were statistical differences between eutopic and normal endometrium with regards to the methylation status and protein levels of RASSF1A. In addition, DNA methylation was observed in eutopic and ectopic endometrium of EMS. Eutopic endometrium is readily available and gene alteration in the eutopic endometrium can be easily detected. Identification EMS-related genes in eutopic endometrium will further reveal the pathogenesis of EMS and offer the basis for targeted gene diagnosis and therapy of EMS (20). Assessment of RASSF1A methylation status in eutopic endometrium of EMS may be a potentially useful biomarker to enhance the early detection of EMS.

It has previously been reported that hypermethylation of RASSF1A is associated with advanced tumor stage in bladder, esophageal, stomach and endometrial cancer $(6,21-23)$. The severity of endometriosis has been clinically classified by numerous systems. The revised classification system of the American Society for Reproductive Medicine, currently widely used, consists of 4 stages (I-IV), with stage III and IV representing more serious cases (12). In the present study, comparing patients with early clinical stage to patients with advanced clinical stage there were statistical differences with regards to RASSF1A methylation in ectopic endometrium. However no statistically significant differences were observed in eutopic endometrium, which suggests that the methylation status of RASSF1A in the EMS eutopic endometrium tissues would not alter with clinical stage; promoter methylation of RASSF1A in the ectopic endometrium may therefore serve a more prominent role in the development and progression of EMS. This is a noteworthy topic that may merit further study.

Transient treatment of cells with demethylating agents can lead to a long-lasting demethylation effect (24). The epigenetic mechanisms represent a heritable dynamic and reversible means of modulating gene expression. The reversible nature of epigenetic aberrations is an important characteristic, since it allows us to search for appropriate pharmacological treatments, or 'epigenetic therapies' (25). In a number of studies it was demonstrated that RASSF1A expression was significantly increased or markedly upregulated in a number of types of cancer, including ovarian cancer and endometrial carcinoma, after treatment with 5-aza-DC (26-28). Therefore, DNA demethylation agents may be used in the future to restore methylation aberrations of RASSF1A in EMS.

However, how methylation of the RASSF1A promoter results in a reduction of protein expression in EMS needs further study. Further studies are necessary to confirm the findings in larger samples and to investigate the promoter in vivo prior to and following 5-aza-DC treatment.

In summary, the present study demonstrated, to the best of our knowledge, for the first time that the high frequency of promoter hypermethylation of the RASSF1A gene contributes to the loss or absent of protein expression in EMS. The results indicated that hypermethylation of the RASSF1A CpG island was involved in the pathogenesis of EMS. This also provides another piece of evidence that EMS may be ultimately an epigenetic disease. This may open up a novel avenue for the development of therapeutics for EMS and provide a potentially promising way for epigenetic reprogramming that restores dysregulated gene expression.

\section{Acknowledgements}

The Central Laboratory of Liaocheng People's Hospital is thanked for providing technical support for the experimental process. The authors would also like to thank all the women who participated in the study. 


\section{References}

1. Giudice LC and Kao LC: Endometriosis. Lancet 364: 1789-1799, 2004.

2. Farquhar C: Endometriosis. BMJ 334: 249-253, 2007.

3. Sampson JA: Ovarian hematomas of endometrial type (perforating hemorrhagic cysts of the ovary) and implantation adenomas of endometrial type. Boston Med Surg J 186: 445-456, 1922.

4. Dammann R, Yang G and Pfeifer GP: Hypermethylation of the cpG island of Ras association domain family 1A(RASSF1A), a putative tumor suppressor gene from the $3 \mathrm{p} 21.3$ locus, occurs in a large percentage of human breast cancers. Cancer Res 61 : 3105-3109, 2001.

5. Agathanggelou A, Honorio S, Macartney DP, et al: Methylation associated inactivation of RASSF1A from region 3 p21.3 in lung, breast and ovarian tumours. Oncogene 20: 1509-1518, 2001.

6. Jo H, Kim JW, Kang GH, et al: Association of promoter hypermethylation of the RASSF1A gene with prognostic parameters in endometrial cancer. Oncol Res 16: 205-209, 2006.

7. Kuzmin I, Liu L, Dammann R, et al: Inactivation of RAS association domain family $1 \mathrm{~A}$ gene in cervical carcinomas and the role of human papillomavirus infection. Cancer Res 63: 1888-1893, 2003.

8. Burbee DG, Forgacs E, Zöchbauer-Müller S, et al: Epigenetic inactivation of RASSF1A in lung and breast cancers and malignant phenotype suppression. J Natl Cancer Inst 93: 691-699, 2001.

9. Starzinski-Powitz A, Gaetje R, Zeitvogel A, et al: Tracing cellular and molecular mechanisms involved in endometriosis. Hum Reprod Update 4: 724-729, 1998.

10. Wu Y, Strawn E, Basir Z, Halverson G and Guo SW: Promoter hypermethylation of progesterone receptor isoform B (PR-B) in endometriosis. Epigenetics 1: 106-111, 2006.

11. Wu Y, Strawn E, Basir Z, Halverson G and Guo SW: Aberrant expression of deoxyribonucleic acid methyltransferases DNMT1, DNMT3A, and DNMT3B in women with endometriosis. Fertil Steril 87: 24-32, 2007.

12. Canis M, Donnez JG, Guzick DS, et al: Revised American Society for Reproductive Medicine classification of endometriosis:1996. Fertil Steril 67: 817-821, 1997.

13. Darehdori AS, Dastjerdi MN, Dahim H, et al: Lack of significance of the BRCA2 promoter methylation status in different genotypes of the MTHFR a1298c polymorphism in ovarian cancer cases in Iran. Asian Pac J Cancer Prev 13: 1833-1836, 2012.

14. Wu Y, Zhang X, Lin L, et al: Aberrant methylation of RASSF2A in tumors and plasma of patients with epithelial ovarian cancer. Asian Pac J Cancer Prev 15: 1171-1176, 2014.
15. Feinberg AP: Phenotypic plasticity and the epigenetics of human disease. Nature 447: 433-440, 2007.

16. Feinberg AP: Epigenetics at the epicenter of modern medicine. JAMA 299: 1345-1350, 2008.

17. Monte NM, Webster KA, Neuberg D, Dressler GR and Mutter GL: Joint loss of PAX2 and PTEN expression in endometrial precancers and cancer. Cancer Res 70: 6225-6232, 2010.

18. Zhang H, Zhao X, Liu S, Li J, Wen Z and Li M: 17 betaE2 promotes cell proliferation in endometriosis by decreasing PTEN via NFkappaB-dependent pathway. Mol Cell Endocrinol 317: 31-43, 2010.

19. Pallarés J, Velasco A, Eritja N, et al: Promoter hypermethylation and reduced expression of RASSF1A are frequent molecular alterations of endometrial carcinoma. Mod Pathol 21: 691-699, 2008.

20. Wang D, Chen Q, Zhang C, Ren F and Li T: DNA hypomethylation of the COX-2 gene promoter is associated with up-regulation of its mRNA expression in eutopic endometrium of endometriosis. Eur J Med Res 17: 1-6, 2012.

21. Byun DS, Lee MG, Chae KS, Ryu BG and Chi SG: Frequent epigenetic inactivation of RASSF1A by aberrant promoter hypermethylation in human gastric adenocarcinoma. Cancer Res 61: 7034-7038, 2001.

22. Maruyama R, Toyooka S, Toyooka KO, et al: Aberrant promoter methylation profile of bladder cancer and its relationship to clinicopathological features. Cancer Res 61: 8659-8663, 2001.

23. Kuroki T, Trapasso F, Yendamuri S, et al: Promoter hypermethylation of RASSF1A in esophageal squamous cell carcinoma. Clin Cancer Res 9: 1441-1445, 2003.

24. Egger G, Liang G, Aparicio A and Jones PA: Epigenetics in human disease and prospects for epigenetic therapy. Nature 429: 457-463, 2004.

25. Nasu K, Kawano Y, Tsukamoto Y, et al: Aberrant DNA methylation status of endometriosis: Epigenetics as the pathogenesis, biomarker and therapeutic target. J Obstet Gynaecol Res 37: 683-695, 2011.

26. Balch C, Yan P, Craft T, et al: Antimitogenic and chemosensitizing effects of the methylation inhibitor zebularine in ovarian cancer. Mol Cancer Ther 4: 1505-1514, 2005.

27. Kang S, Lee JM, Jeon ES, et al: RASSF1A hepermethylation and its inverse correlation with BRAF and/or KRAS mutations in MSI-associated endometrial carcinoma. Int J Cancer 119: 1316-1321, 2006.

28. Donninger H, Vos MD and Clark GJ: The RASSF1A tumor suppressor. J Cell Sci 120: 3163-3172, 2007. 